\title{
Feedback en educación médica
}

\author{
J.M. Fornells ${ }^{a}$, X. Julià b ${ }^{\text {, J. Arnau }}{ }^{\text {a }}$ J.M. Martínez-Carretero a
}

La propuesta de un modelo centrado en la persona que aprende y el desarrollo de estrategias de aprender a aprender persigue que los estudiantes sean más reflexivos y más autónomos en su propio proceso de aprendizaje y que se conviertan en los protagonistas de dicho proceso. Se trata, en definitiva, de dar los elementos necesarios a los estudiantes para que puedan autogestionar un proceso de aprendizaje permanente a lo largo de toda su vida profesional, una cuestión crucial en un contexto de evolución constante de los conocimientos. El feedback sería el retorno de información sobre su proceso de aprendizaje de acuerdo con unos objetivos preestablecidos. El feedback presenta información y no juicio, a diferencia de la evaluación, en consecuencia siempre es formativo. El feedback no es un fin en sí mismo sino un instrumento que informa al estudiante sobre su proceso de aprendizaje y facilita los cambios necesarios. El feedback estructurado y centrado en quien aprende se caracteriza por: autorreflexión del estudiante, centrado en quien aprende, preparación previa y relación de confianza tutor-residente.

Palabras clave. Autoaprendizaje. Evaluación formativa. Feedback.

\section{Feedback in medical education}

The proposal of a learner-centered model and the development of strategies for learning to learn, intends that students are more reflective and more independent in their own learning process and that they become the protagonists of this process. It really tries to give the students the necessary elements so that they can self-manage a process of permanent learning throughout their professional life, a crucial question in a context of constant evolution of the knowledge. The return of information on the process of learning, in agreement with pre-established objectives. Feedback presents/displays information and is non-judgemental, unlike the evaluation.
Feedback always is formative. Feedback is not an aim in itself, but an instrument that informs the student on its process of learning and facilitates the necessary changes. Structured and centered learning feedback is characterized by: student self reflection, centered in the learning person, previously prepared and a confident relationship between tutor and student.

Key words. Feedback. Formative assessment. Self directed learning.

\section{La enseñanza centrada en el que aprende. Aprender a aprender}

Tradicionalmente, el proceso de enseñanzaaprendizaje se caracteriza por estar basado en un modelo centrado en el que enseña, en el cual predomina la transmisión de información del docente al discente y donde se valoran las capacidades memorísticas del estudiante. La propuesta de un modelo centrado en la persona que aprende y el desarrollo de estrategias de aprender a aprender persigue que los estudiantes sean más reflexivos y más autónomos en su propio proceso de aprendizaje y que se conviertan en los protagonistas de dicho proceso.

Se trata en definitiva de dar los elementos necesarios a los estudiantes para que puedan autogestionar un proceso de aprendizaje permanente a lo largo de toda su vida profesional, cuestión crucial en un contexto de evolución constante de los conocimientos y otros elementos que configuran las competencias de los profesionales ante el reto de dar respuesta a las necesidades de salud de la población.

Dentro de esta perspectiva, el protagonista del proceso enseñar-aprender es la persona que a Institut d'Estudis de la Salut.

b Ayuntamiento

de Pineda de Mar España.

E-mail

joma.fornells@

gencat.net

Cuestionario

de acreditación

www.educmed.net 
aprende, ya sea el alumno en el pregrado, el residente en el posgrado o el médico a lo largo de su trayectoria profesional. El alumno (quien aprende) adquiere la condición de participante activo de su propio proceso de aprendizaje.

El aprendizaje autodirigido permite que el alumno sea capaz de saber qué tiene que aprender (objetivos de aprendizaje), cómo debe aprenderlo (metodologías que utilizará) y si consigue los objetivos propuestos mediante la autoevaluación. Para todo esto, el alumno, que ya es un adulto, cuenta con la ayuda, la orientación y el soporte de personas expertas que le acompañan en este camino que ha de llevarle de aprendiz a experto. Y de entre todas estas personas destaca la figura del tutor o mentor que desempeña un papel clave [1].

La primera premisa para que el tutor pueda desarrollar su trabajo y acompañar de forma efectiva al alumno es que exista una relación de confianza entre ambos que será fundamental para que se pueda desarrollar un proceso de evaluación formativa.

Recordemos que la evaluación formativa tiene como finalidad detectar los puntos fuertes y los débiles de la persona que aprende respecto a unos objetivos y unas competencias claramente definidas, y si es necesario proponer medidas correctoras.

Ya hemos dicho que la relación de confianza es clave para este trabajo conjunto entre alumno y tutor. También lo es que se dé en un marco seguro para el alumno, que favorece una reflexión personal sobre lo que le sucede en este recorrido que le ha de llevar a ser un médico o un especialista.

\section{El feedback estructurado y centrado en la persona que aprende}

El concepto de feedback, información usada por un sistema para hacer ajustes en la consecución de un objetivo, fue ampliado por Weiner [2] hacia un ámbito más humanístico: feedback es el control de un sistema mediante la reinserción en el propio sistema de los resultados de su actuación. Si esta información es capaz de producir cambios en el método general y en el de actuación, se trata ya de un proceso que se puede denominar aprendizaje.

Si se aplica a estudiantes, el feedback sería el retorno de información sobre su proceso de aprendizaje de acuerdo con unos objetivos pre- establecidos. En la bibliogarfía vemos que la palabra feedback se utiliza para referirse a situaciones muy diferentes ('dar feedback', 'recibir feedback', 'feedback unidireccional', etc.). Por ello es necesario diferenciar los tipos de feedback empleados de forma más común:

- Feedback informal: es aquel que se produce inmediatamente después de una actividad de aprendizaje. No hay espacio para la reflexión, y se hace de forma inmediata.

- Feedback formal: se pacta el momento en que se hará el feedback, hay espacio para la reflexión sobre un tema concreto y con una duración pactada.

- Feedback individual: se produce entre un alumno y su tutor o un docente. También se podría dar el caso de feedback entre un residente, por ejemplo, y un estudiante de medicina o entre dos colegas. Este feedback puede ser presencial o bien escrito.

- Feedback grupal: se da entre un grupo de alumnos de un mismo nivel (curso, año de residencia) y el tutor, que suele actuar como facilitador. El feedback es multidireccional y requiere una cierta habilidad en la dinámica de grupos.

- Feedback máquina-persona: es el que se produce mediante ordenadores o maniquíes, por ejemplo.

En la práctica se pueden combinar diferentes formas de feedback, por ejemplo, feedback individual y formal.

Sin embargo, muchas veces, hay una cierta confusión que envuelve al concepto de feedback. Ende [3] expone que los términos feedback y evaluación son empleados a menudo de manera intercambiable, pero el primero presenta información y no juicio, a diferencia de la evaluación. El feedback siempre es formativo, mientras que cuando hablamos de evaluación dentro de este contexto es sumativa.

El hecho de que el alumno entienda el carácter formativo del feedback es clave para su éxito. Si éste percibe el feedback como una forma de evaluación sumativa generará reacciones defensivas y el feedback perderá su efectividad.

El feedback no es un fin en sí mismo, sino un instrumento que informa al estudiante sobre su proceso de aprendizaje y facilita los cambios necesarios. De la misma manera puede ser una herramienta muy importante que indica cómo se 
desarrolla un programa educativo hacia la consecución de sus objetivos.

Así pues, podemos concluir que hay diferentes maneras de llevar a cabo el feedback y que la efectividad no será la misma. Por lo tanto, es necesario que nos refiramos aquí al feedback estructurado y centrado en quien aprende.

Algunas de las características que pueden hacer que el feedback sea más efectivo son según Ende [3] las siguientes:

- La relación de confianza entre discente y docente. El estudiante no ha de ver a la persona que le da feedback como alguien que le juzga y le evalúa.

- El feedback ha de ser planificado y realizado en el tiempo oportuno, es decir, suficientemente cerca de las situaciones que se quieren analizar.

- El feedback ha de estar basado en datos y a poder ser en la observación directa. Habrá veces en que el tutor o responsable del estudiante se guiará por informaciones que le lleguen de otras fuentes.

- El feedback es descriptivo y no evaluativo. Hemos de tener cuidado con el lenguaje que utilizamos.

- Se valoran las competencias y las actuaciones profesionales, no a personas. Un estudiante puede tener un rendimiento excelente en una determinada área y ser deficiente en otra.

Según el mismo autor, sin feedback los estudiantes tienen poca información sobre sus puntos fuertes y débiles, los errores no se corrigen, la buena praxis no se refuerza y la competencia clínica se adquiere de manera empírica o no se adquiere.

El feedback estructurado de la manera más convencional, tal como lo preconizan Ende [3], Silverman [4,5], Pendlenton [6] o Katz [7], tiene los rasgos básicos siguientes:

- Los temas que se abordan han de ser concretos y pactados previamente.

- El día y la hora también han de ser pactados, con reflexión previa.

- Primero hay que tratar los puntos positivos y después los puntos que se deben mejorar y las medidas correctoras, si es preciso.

- En primer lugar opina el alumno y, después, el tutor valida.

- Las conclusiones han de ser explícitas.
Otros autores como Kurtz [8] propugnan una mayor flexibilidad a la hora de aplicar el esquema del feedback convencional, ser menos rígido y dar más el protagonismo de la sesión al estudiante. Se trata de conseguir un equilibrio entre los puntos fuertes y los puntos que hay que mejorar, ser más eficiente en términos de tiempo consumido y dar prioridad a aquello que más preocupe al residente o estudiante.

El feedback puede ocurrir en varios entornos de aprendizaje, como pueden ser prácticas clínicas o aprendizaje basado en problemas en el grado o las rotaciones del residente o temas sugeridos por él mismo en la formación especializada. En cada uno de estos contextos el tutor o docente deberá considerar la adecuación de las características generales descritas.

Asimismo, el feedback se puede plantear asociado a instrumentos concretos de evaluación, por lo general formativa. De esta manera, el mini-CEX (Clinical Evaluation Exercise) lleva aparejado, después de la observación estructurada de un encuentro clínico, una sesión de feedback entre el docente-observador y el estudiante [12]. Lo mismo sucede con la técnica de $360^{\circ}$, en la que se produce un feedback a partir de varias fuentes [13]. Recientemente el feedback es una pieza clave de los distintos instrumentos de evaluación aplicados en el Foundation Programme [14].

\section{Búsqueda sobre el feedback: la revisión sistemática del BEME (best evidence in medical education)}

En 1999 se constituyó la Best Evidence Medical Education Collaboration (BEME) [9], bajo los auspicios de la Asociación para la Educación Médica en Europa (AEME, en inglés AMEE). El objetivo del BEME es seleccionar las aportaciones metodológicas existentes en el ámbito de la educación médica para identificar sus posibles limitaciones o carencias y proponer futuras líneas de trabajo para optimizar la eficacia y la eficiencia de las intervenciones educativas disponibles o propuestas. El método de trabajo consiste en la revisión sistemática de la bibliografía publicada sobre un tema por parte de un grupo de trabajo constituido para tal efecto. Tanto el tema como el grupo deben ser previamente aprobados por la secretaría del BEME, sita en el Centro para la 
Educación Médica de la Universidad de Dundee (Escocia, Reino Unido).

El Institut d'Estudis de la Salut (IES) decidió promover la constitución de un grupo en Barcelona con la doble intención de colaborar con el BEME y al mismo tiempo fomentar en nuestro país la educación médica basada en la evidencia.

El tema escogido fue el uso del feedback en la evaluación (feedback in assessment). El IES invitó a todos los sectores de la educación médica (medicina, odontología, enfermería, puericultura, etc.) a participar en el proyecto, además de buscar la implicación de tutores y responsables docentes del mundo universitario. También se contó con la colaboración de algunos expertos extranjeros.

Algunas de las preguntas que surgieron de las primeras reuniones de trabajo realizadas fueron:

- ¿Qué tipos de feedback (individual, grupal, presencial) son más efectivos?

- ¿Cuáles son las características que hacen más efectivo al feedback? ¿Estructurado? ¿En qué medida? ¿Influye el nivel de ortodoxia o de heterodoxia?

- ¿El uso de un determinado modelo de feedback mejora el nivel de autoconocimiento de los alumnos respecto a su proceso de aprendizaje?

- ¿El feedback es útil y efectivo para la mejora de las habilidades docentes del tutor?

El grupo de trabajo del BEME en Barcelona [10] celebró en abril de 2002 sus sesiones iniciales de trabajo para elaborar el protocolo que tenía que servir de base y de punto de partida. Se definió el título exacto de la pregunta sobre la que trabajaba el grupo, además de los objetivos, los criterios de inclusión y exclusión, y los criterios y estrategias de búsqueda de los artículos [11]:

- El título fue: 'El impacto del feedback en la evaluación formativa y sumativa y su efectividad en la mejora del conocimiento, de las habilidades y las actitudes de los estudiantes de ciencias de la salud durante el proceso de aprendizaje, y en las habilidades de enseñanza de los tutores'.

- El objetivo de la pregunta fue establecer cuáles son las características del feedback realizado por el profesorado que sigue la evaluación formativa y sumativa, sistema que permite a los estudiantes de ciencias de la salud, tanto de pregrado como de posgrado, mejorar el co-
Tabla I. Criterios de inclusión.

Tema

- Criterios de inclusión

Estudiantes

- Pregrado

- Posgrado

- Estudiantes de ciencias de la salud

Tutores

- Personal docente

- Tutores

Evaluación hecha por

- Tutor personal

- Tutor clínico

- Profesor

- Estudiantes de ciencias de la salud

Educación

- Título académico

Propósito del feedback

- Formativo

- Sumativo

Método del feedback

- Estructurado

- Formal

- Discusión cara a cara

- Escrito

Tiempo del feedback

- Continuo

- Concluyente

Resultados

Cambios en:

- Conocimientos

- Habilidades clínicas

- Habilidades en enseñanza

- Actitud

- Competencia

- Autoaprendizaje

- Rendimiento

nocimiento, las habilidades y las actitudes de forma más efectiva, y que permite a los tutores perfeccionar las habilidades de enseñanza.

- Los criterios de inclusión estaban resumidos en la tabla I. 


\section{Tabla II. Estrategia de la búsqueda.}

\section{Tipos de bibliografía}

- Revistas de ciencias de la salud y de educación

- Literatura gris (documentación no indexada)

- Libros de ponencias

- Tesis

Idiomas

- Inglés

- Catalán

- Castellano

- Francés

- Alemán

Bases de datos

- Medline

- CINAHL

\section{Años cubiertos}

- 10 últimos años (desde noviembre de 1992)

\section{Palabras clave}

- Evaluación formativa

- Evaluación sumativa

- Feedback y evaluación o método de enseñanza

- Pregrado, posgrado o educación médica

- Médicos, enfermeras, puericultoras o dentistas

- Tutores

- Estructurado o planeado

- Competencias clínicas

- Efectividad

- Aprendizaje

- Educación

- Habilidades de enseñanza

- Como criterios de exclusión se consideraron la enseñanza continuada y el feedback realizado de manera informal o con simulación (pacientes simulados o maniquíes).

- La estrategia de búsqueda fue resumida en la tabla II.

El proceso de revisión se realizó en diferentes etapas y alcanzó la bibliografía publicada hasta junio de 2005. Se hizo un seguimiento manual de las ediciones de Medical Education y Medical Teacher hasta diciembre de 2006, por ser las dos publicaciones que proporcionaron mayor número de artículos.

Se revisaron unos 2.000 artículos y referencias, de los cuales sólo unos 25 mostraron resultados claros y verdaderos (clear and likely to be true). Por lo tanto, la primera conclusión fue que hay una falta de estudios que demuestren con evidencia científica que el uso del feedback en la evaluación proporciona mejores resultados que si no se utiliza este recurso educativo, más allá de la simple descripción.

Características mayoritarias del feedback descrito en los artículos revisados:

- Feedback proporcionado por tutores o educadores.

- Recibido por estudiantes/residentes.

- Feedback individual y unidireccional.

- Feedback oral y escrito en un porcentaje parecido de casos. Destaca la todavía poca descripción del uso de nuevas tecnologías.

- Feedback que contiene un proceso de metodología y conocimiento.

- El tipo de evaluación más descrito es la observación.

Emplazamos a la lectura del informe completo del que se tiene prevista su publicación próximamente por los canales habituales que utiliza la Colaboración BEME [9].

\section{Bibliografía}

1. Murray E. Challenges in educational research. Med Educ 2002; 36: 110-12.

2. Weiner $\mathrm{N}$. The human use of human being, in cybernetics and society. Boston: Houghton Mifflin; 1950. p. 71.

3. Ende J. Feedback in clinical medical education. JAMA 1983; 250: 777-81.

4. Silverman J, Drapper J, Kurtz S. The Calgary-Cambridge approach to communication skills teaching II: the SET-GO method of descriptive feedback. Education for General Practice 1997; 8: 16-23.

5. Silverman J, Draper J, Kurtz S. The Calgary-Cambridge approach to communication skills teaching I: agenda-led outcome based analyses of the consultation. Journal in Education for General Practice 1996; 7: 288-99.

6. Pendlenton D, Tate P, Havelock P. The consultation: an approach to learning and teaching. Oxford: Oxford University Press; 1984.

7. Katz PO. Providing feedback. Gastrointest Endosc Clin N Am 1995; 5: 347-55.

8. Kurtz SM. Curriculum structuring to enhance commu- 
nication skills development. In Steward M, Roter D, eds. Communicating with medical patients. Newbury Park, CA: Sage Publications; 1989.

9. Colaboración BEME (Best Evidence Medical Education). URL: http://www.bemecollaboration.org. [22.02.08].

10. Centeno A, Martínez-Carretero JM. Innovaciones, investigación y evidencias en educación médica. Colaboración EMBE (Educación Médica Basada en la Evidencia). Educ Med 2003; 6: 26-30.

11. Julià X, Fornells JM, Arnau J, Martínez-Carretero JM. Leducació mèdica basada en l'evidència. Col-laboració BEME/EMBE. Ann Med Intern 2002; 85: 192-5.
12. Holmboe ES, Yepes M, Williams F, et al. Feedback and the Mini Clinical Evaluation Exercise. J Gen Intern Med 2004; 19: 558-61.

13. Sargeant J, Mann K, Ferrier S. Exploring family physicians' reactions to multisource feedback: perceptions of credibility and usefulness. Med Edu 2005; 39: 497-504.

14. Carr S. The Foundation Programme assessment tools: an opportunity to enhance feedback to trainees? Postgrad Med J 2006; 82: 576-79. 